\title{
Cognitive Intervention Protocol for Age-Related Memory Impairments
}

\author{
Ana Maria Portugal ${ }^{1,2}$, Daniela Silva Ferreira ${ }^{1,2}$, Joana \\ Santos Reis ${ }^{1,2}$, Francisco Pinho ${ }^{3}$ \\ ${ }^{1}$ Life and Health Sciences Research Institute (ICVS) \\ School of Health Sciences, University of Minho \\ 4710-057 Braga, Portugal \\ ${ }^{2}$ ICVS/3B's - PT Government Associate Laboratory \\ Braga/Guimarães, Portugal \\ ${ }^{3}$ Department of Industrial Electronics, University of Minho - \\ Campus de Azurém, 4800-058 Guimarães, Portugal \\ amsilva@ecsaude.uminho.pt, ex3046@ecsaude.uminho.pt, \\ joanareis@ecsaude.uminho.pt, id2712@alunos.uminho.pt
}

Abstract- With the number of elderly people increasing tremendously worldwide, comes the need for effective methods to maintain or improve older adults' cognitive performance. Using continuous neurofeedback, through the use of EEG techniques, people can learn how to train and alter their brain electrical activity. A software platform that puts together the proposed rehabilitation methodology has been developed: a digital game protocol that supports neurofeedback training of alpha and theta rhythms, by reading the EEG activity and presenting it back to the subject, interleaved with neurocognitive tasks such as n-Back and Corsi Block-Tapping. This tool will be used as a potential rehabilitative platform for age-related memory impairments.

Keywords - Neurofeedback; EEG; Cognitive intervention; Healthy aging; Serious Games; Brain-Computer Interface, Alpha, Theta, Memory.

\section{INTRODUCTION}

With the growing life expectancy, the number of elderly people is increasing tremendously worldwide. As a consequence, the burden of age-associated disorders, such as Alzheimer and other kinds of dementia, is also exponentially growing, affecting about $50 \%$ of all elderly patients with a high cost to society and a major impact on family and caregivers [1]. Impairment of cognitive ability is associated with a progressive decrease of synaptic plasticity and neuronal inter-connectivity, which are neurophysiological characteristics of the aging brain [2]. Neuroplasticity is characterized by neural redundancy and plastic remodeling of brain networking, that can be secondary to mental or physical training.

Cognitive processes like executive and associative functions are commonly trained on behavioral neurocognitive (NC) tasks, since it has been reported their effectiveness and durability on trained and near transfer tasks [3]. Some studies have described the functional benefits of cognitive training (memory, reasoning, speed of processing) in the elderly population [4], and more specifically, studies on healthy aging have been providing growing evidence on the improvement or maintenance of cognitive abilities when targeting memory domain $[5,6]$.

\author{
Nuno S. Dias ${ }^{1,2,4}$ \\ ${ }^{1}$ Life and Health Sciences Research Institute (ICVS) \\ School of Health Sciences, University of Minho \\ 4710-057 Braga, Portugal \\ ${ }^{2}$ ICVS/3B's - PT Government Associate Laboratory \\ Braga/Guimarães, Portugal \\ ${ }^{4}$ DIGARC, Polytechnic Institute of Cavado and Ave, 4750- \\ 810 Barcelos, Portugal \\ nunodias@ecsaude.uminho.pt
}

However, these studies also showed poor results on transferability of tasks for real-life activities and suggest combination with other approaches to reach higher levels of rehabilitation, like cognitively, physically or socially stimulating activities of everyday life [3]. Additionally, novel tools to guide training strategies, as neuroimaging techniques, seem to be a promising field.

In this context, recent studies in patients of Alzheimer Disease [7] and mild cognitive impairment [2] suggest that patients' temporal and spectral EEG features significantly differ from healthy subjects'. Simultaneously, noticeable attention has been paid to Brain-Computer Interface (BCI) applications that address the functional recovery of the central nervous system to improve the cognitive and behavioral performance of children with attention deficits [8], enhance memory retrieval [9] and reduce pain perception [10]. These studies employ neurofeedback (NF) training on their protocols, where the subject is instructed to modulate his own brain rhythms whose feedback is provided by a BCI. Although these studies demonstrate an application context significantly different from the classic BCIs [11], the NF-oriented BCI perspective has been suggested as a promising tool to enhance plasticity and able to provide new outcomes for cognitive functional recovery [12]. Based on real-time EEG recordings, its processing and classification, NF BCI protocols might be able to guide neuroplasticity to promote recovery of brain functions.

The development of a therapeutic tool that stimulates neuronal plasticity mechanisms in individuals with cognitive deficits resultant from brain aging is being pursued. The neurorehabilitation plan combines NF training [13] with common NC tasks (working memory), in a training protocol that interleaves trials of both approaches. This intervention strategy relies on an operant conditioning $\mathrm{BCI}$ that prompts the subject to modulate a particular rhythm of the EEG in order to improve performance on NC tasks. In contrast with either NC or NF training single-methodology, we believe that the complementary methodology affords further rehabilitation potential by providing self contained feedback to the subject: 
NF training provides the current and target cognitive states and $\mathrm{NC}$ training provides the accuracy on test performance addressing specific cognitive functions. Besides promoting larger functional recovery, this combined approach may also increase transfer and durability effects of the training.

\section{BACKGROUND}

Performance-related electroencephalogram (EEG) markers are currently being studied in the group, by acquiring EEG data during the execution of the Wisconsin Card Sorting Test (WCST) in elder subjects.

EEG power, ERD/ERS (event related desynchronization/synchronization) [14] and coherence, were evaluated and correlated with performance measures. Preliminary results suggest that these EEG features may be regarded as phenotypes of good performance on memory and executive function tasks. These 'phenotypes' may be useful for the developing of therapeutic tools for age-related cognitive impairment using NF and will be published somewhere else.

The selection and identification of EEG phenotypes is deeply important in the design and outcome assessment of neurofeedback, since they will be guiding the neurofeedback protocol design and indicating the target "EEG phenotype" to pursue during cognitive intervention.

\section{REHABILITATION PLATFORM AND NEUROFEEDBACK TRAINING PROTOCOL}

The software system that put together the proposed rehabilitation platform includes: a digital game protocol that presents to the user his/her brain state in a simple way, and stimulate him/her to improve. This tool will be used as a rehabilitative platform for neurofeedback (NF). The platform has the structure of a NF-oriented BCI -acquisition and storage of EEG signals in real-time, followed by processing and translation of the signals. This classification is presented to the user, in a way of promoting him to modulate his signals, and then a new cycle begins (Fig. 1) [15].

Additionally, the rehabilitation platform includes a set of neurocognitive tasks. In the same way, there will be acquisition and storage of the EEG signals, during the course of the tasks; however, in this case, the user will not receive any feedback from the processing and translational algorithms.

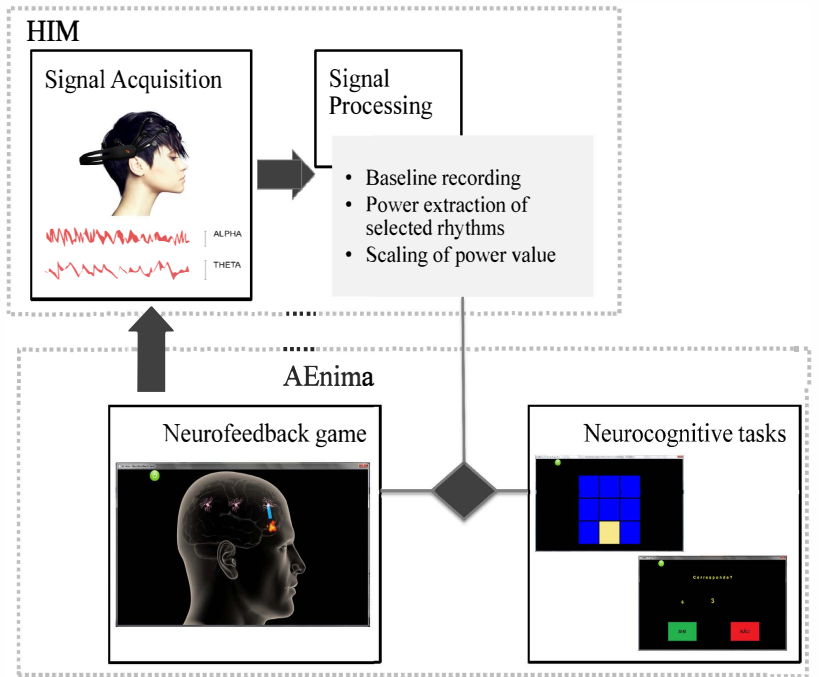

Figure 1. Diagram of the proposed rehabilitation platform, that includes neurofeedback and neurocognitive training.

\section{A. $\mathrm{BCI}++$}

A BCI platform supports the NF training by reading the EEG activity and presenting it back to the subject. For this propose it will be used a software developed by Sensibilab, the $\mathrm{BCI}++$ platform [16]. $\mathrm{BCI}++$ is divided in two main modules interconnected: a first one called HIM, oriented for the realtime acquisition and processing of the EEG signals and the translational algorithm, and a second one called AEnima who is responsible for providing visual or auditory feedback about the current brain state of the subject, in a form of a digital game.

\section{B. Translational Algorithm}

The central piece of a BCI is the translational algorithm implemented. It has to combine the EEG features from various electrodes and translate them into a brain state representation, allowing a classification in real-time of the subject's current condition compared with the target one.

In the proposed algorithm, the focus will be on two brain rhythms, theta and alpha, that are crucial on memory-related NF protocols, [17, 18]. The power of theta and alpha rhythms is calculated on $1 \mathrm{~s}$ segments and is updated 10 times per second. In the beginning of each session, a baseline record will be performed during $60 \mathrm{~s}$. The subject has to be as relaxed as possible, while being exposed to the graphic environment of the game (Fig. 2). The medium and standard deviation of values of power in this segment will be the parameters of a $\mathrm{z}$ score for the classification algorithm. Accordingly with the level of difficulty, the minimum and maximum values will be $2 \mathrm{x}, 3 \mathrm{x}$ or $4 \mathrm{x}$ the standard deviation. Every time the algorithm is updated, the power values will be scaled on the new range defined by new minima and maxima values.

Within each difficulty level, the values for minimum and maximum power can also be re-evaluated to be more adequate to the user performance. So, every time the user reaches a value higher than the maximum defined or a value lower than the minimum defined, these values will became the actual maximum and minimum values. 
In order to adjust the game difficulty level, and consequently, the minimum-maximum range, the proposed algorithm evaluates regularly the latest power values, by raising or decreasing the game difficulty when the power $\mathrm{z}$ score is near the extreme values.

\section{Neurofeedback Game}

The neurofeedback game is being developed in AEnima which is based on the Irrlicht game engine . The game is subdivided in two game modes: one where the feedback is calculated from the theta rhythm power; and another one where the feedback is calculated from alpha power. Essentially it will accept the feedback value of the power for one of the rhythms from the translation algorithm, and presenting it to the player, in a form of a bar whose length increases or decreases depending on the power value. The higher the value of the power, the better the subject performance.

The game is designed with a therapeutic meaning: the goal of the game is to extinguish a fictional fire, either nearby the hippocampus (theta NF) or in the prefrontal cortex (alpha NF), with a neuron that carries an axon full of water that has to reach the fire region. This axon is animated as a bar that increases or decreases its length (approaching or withdrawing of the fire focus) according to alpha or theta power, calculated on the translational algorithm (Fig. 2). The sum of the power values yield during the game will set the score of the player. The fire in the hippocampus will also diminish as the bar increases towards it (and the score increases), and will be completely extinguished if the bar reaches maximum value.

Additionally, the adjustment of the difficulty level of the game by the algorithm is represented by a change in the location of the fireman neuron, for a further region in the case of a level upgrade. The power values range will be larger, and it will be more difficult for the player to reach the fire.

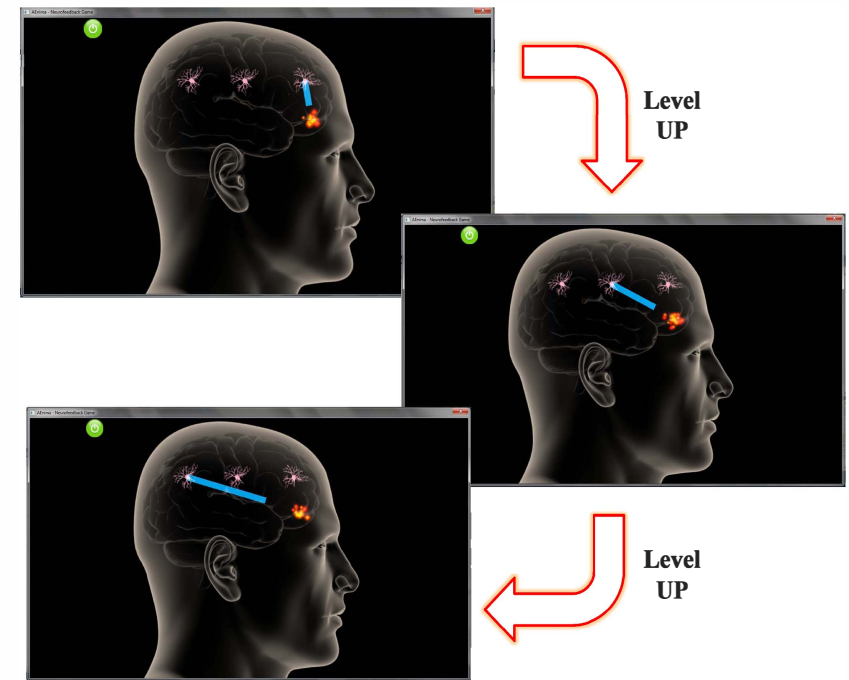

Figure 2. Representative images of the Neurofeedback game implemented in AEnima. There is a fire in the hippocampus region and the player has to increase the blue bar, that starts in the neuron, to reach this fire and extinguish it. Three levels of difficulty are presented here, that vary in the distance from the neuron to the fire.

\section{Neurocognitive Tasks}

Two different neurocognitive tasks, both assessing working memory domain, were implemented. The cognitive tests that will be used are the Corsi Block-Tapping Test, adapted from PEBL Psychological Test Battery [19], and the N-back Test, both implemented in the AEnima game protocol. They will not receive any feedback or classification from $H I M$ and the translational algorithm.

1) Corsi Block-Tapping Test (forward and backward): is a classic test to assess visual-spatial short term working memory. The player has to track a sequence of up to nine identical blocks being highlighted and reproduce it, either in forward or backward order (Fig. 3).

The sequence starts with only two blocks, but when the player repeats correctly one sequence, the game upgrades the level, increasing the number of blocks in the next sequence. On the other hand when the subject repeats wrongly in one sequence, the game downgrades the level and decreases the number of blocks in the next sequence. At the end, measures of block span, accuracy, reaction time and mean of number of blocks of the sequence are computed, as trial reports for every game block performed.

The game will start with the lower difficulty level, which is the forward Corsi task, and after 2 blocks of the game it will shift to the backward Corsi task.
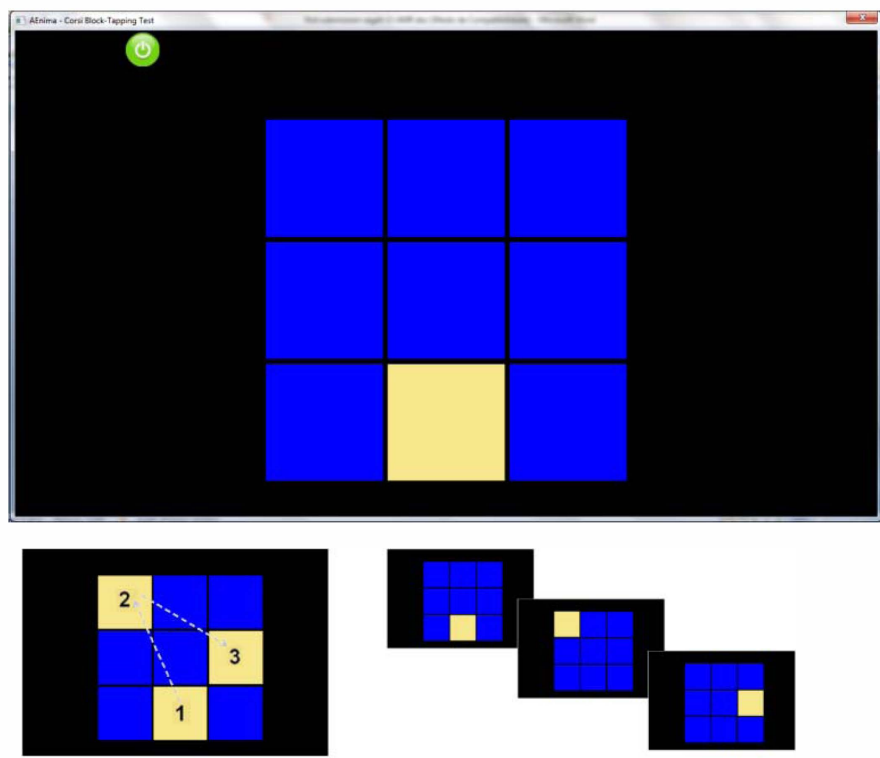

Figure 3. Representative image of the Corsi Block-Tapping Task implemented in AEnima. There are 9 blue blocks and a sequence of them is presented to the player, who has to reproduce it. When the player reproduces correctly, the number of the next sequence is increased (up to nine), and when it reproduces it incorrectly the number is decreased.

2) N-Back Test: is a commonly accepted task to measure cognitive performance in working memory domain. The subject is presented successively with digits and has to indicate whether or not the current digit matches the $n$ instances before (1-back - the previous digit; 2-back - the digit that appeared two times earlier) (Fig. 4).

${ }^{1}$ irrlicht.sourceforge.net 
The game will start with the lower dificulty level, which is the 1-back task. The subject only has to evaluate if the current digit is the same as the previous one. Measures of accuracy and reaction time are computed at the end, as trial reports for every block performed. If the subject is able to perform successfully two blocks, then there will be a shift to the 2-back task. In this case, the subject has to remember and compare the current digit to the digit that appeared twice behind. The subject will perform two blocks and then the game stops.

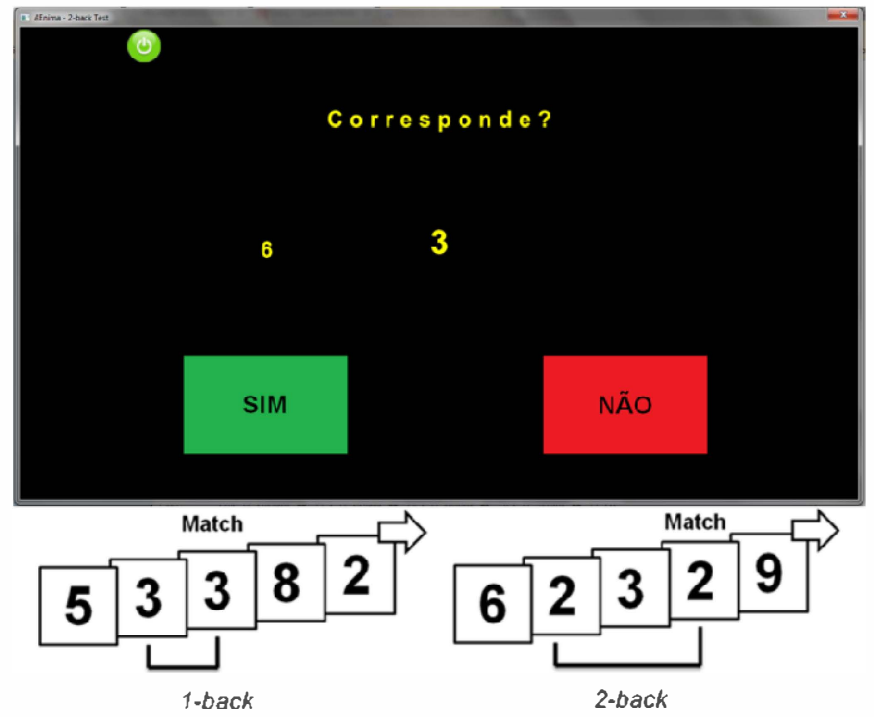

Figure 4. Representative image of the 2-Back Task implemented in AEnima. A digit is consecutively appearing in the screen, and the player has to warn if it matches the digit presented two times earlier in the game.

3) Arrow Flanker Test: Additionally, the Arrow Flanker Test was implemented as an AEnima protocol, for attention domain assessment, and was also adapted from PEBL Psychological Test Battery [19]. In each trial of the game, the subject is presented with a set of arrows. The player has to pay attention to the central target arrow and give a directional response - left or right keys - within a maximum of 1500 milliseconds. The target arrow can be flanked by stimuli in the same direction (congruent), in the opposite direction (incongruent), or not be flanked (neutral) (Fig. 5). Measures of accuracy and reaction time for each stimuli condition are computed in the end of the game, as trial reports for every block performed.

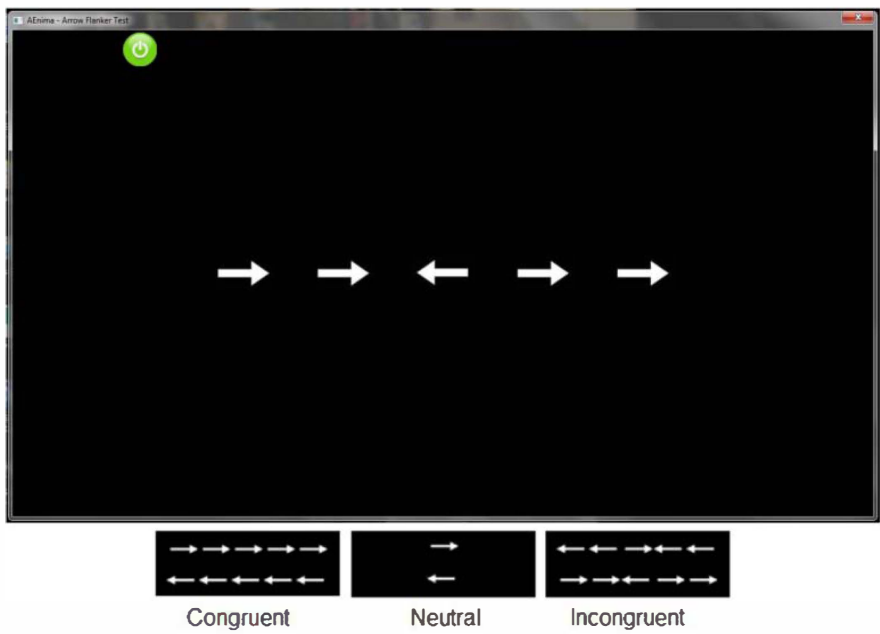

Figure 5. Representative image of the Arrow Flanker Test implemented in AEnima. A set of arrows is presented and the player has to give a directional response to the central arrow (target), in a determined set of time. The type of stiimulus that can flank the target arrow are also represented at the bottom of figure.

\section{COGNITIVE INTERVENTION PROTTOCOL}

A pilot study consisting of a rehabilitative intervention with elder subjects will be performed. These subjects belong to an already existing healthy ageing study cohort containing subjects (males and females) with age above 55 years old, without any diagnosed dementia, disabling pathologies or disease previously tested and known to have average performance on different neuropsychological tests, with respect to memory and executive function (including the Mini-Mental State Examination (MMSE), the Stroop Color and Word Test (Stroop test), the Selective Reminding test (SRT) and the Block Design sub-test of the Wechsler Adult Intelligence Scale) [20].

Depending on the cognitive intervention experienced, subjects will be randomly divided in 4 sets, with 10 subjects each, gender balanced. Statistical power was calculated supporting significant differences with the proposed sample size.

The intervention will took place in Health Care Centers in Guimarães and will be carried on 2 weeks in this way:

TABLE I. DAILY TIME LINE FOR REHABILITATIVE INTERVENTION

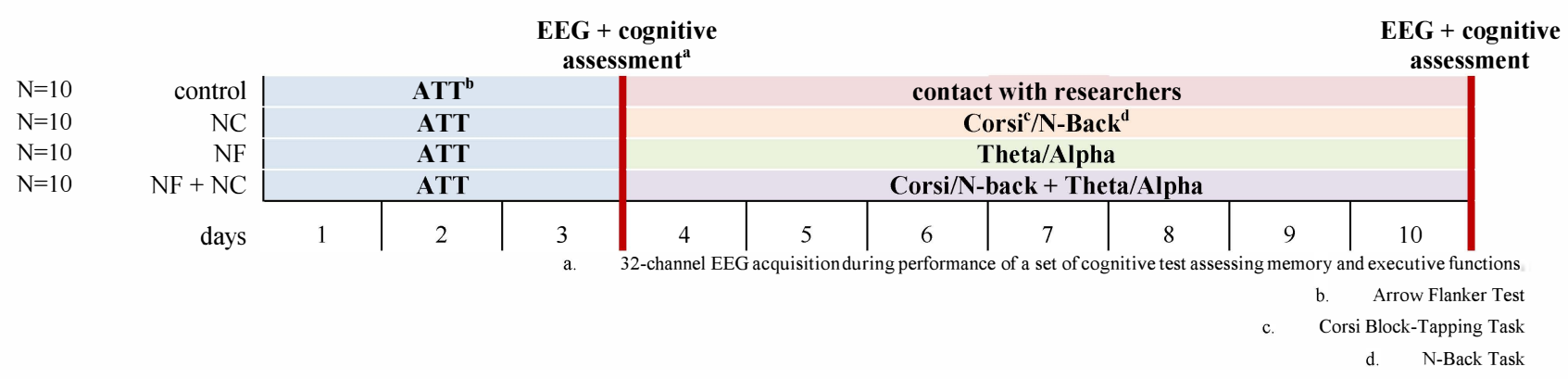


During the training, subjects will be sited in an illuminated and acclimatized room distancing $50-80 \mathrm{~cm}$ from a 17 inch computer screen, with touch technology. They will respond to the neurocognitive tasks using touch screen, except for the Arrow Flanker Test, where they will use keyboard keys.

In the first 3 days all subjects will be submitted to the Arrow Flanker test during 10 minutes (5 blocks of 42 trials). This step aims to guarantee that all the subjects present minimum attention level in order to be able to perform the subsequent tasks. It is a pre-requisite in order to give a baseline from where all the subjects will start, independently from the intervention protocol they will follow.

After the third day, a 32-channel EEG will be acquired from all the subjects while performing a battery of cognitive tests (Stroop task, Trail-making test, Digit Span and Matrix Rotation), that assess predominantly executive function and memory state of the patient. At this time, subjects that did not reach the expected results in attention test will be excluded from the experiment.

During the last seven days the subjects will be submitted to a 30-minute intervention protocol each day, since this training period seems to have effects on behavior and electroencephalogram measures [21-23] accordingly to their experimental group:

- Neurocognitive (NC) experimental group - patients will be submitted to sessions of working-memory tasks: Corsi Block-Tapping (4 blocks of 15 trials) and N-Back (4 blocks of 36 trials).

- Neurofeedback (NF) experimental group - patients will perform sessions of neurofeedback game: modulation of theta or modulation of alpha rhythm, with 8 minutes each game.

- $\quad$ Neurofeedback and neurocognitive $(\mathrm{NF}+\mathrm{NC})$ patients will perform interleaved sessions of a neurocognitive test and a neurofeedback game. The test and the game will change each day.

- Control - subjects will contact with clinicians and researchers, however, they will not perform training at all.

After the 10th day, subjects will repeat the previously described cognitive battery while acquiring EEG, for a posttraining reassessment, in order to document any effects of this intervention. This session is also aimed at acquire a follow-up of the subjects during training time and have feedback of the protocol developed.

The experiments being proposed are already approved by the ethics commission of the cohort of the European Switchbox Project.

\section{FUTURE PERSPECTIVES AND CONCLUSIONS}

In the end of the cognitive intervention, the behavioral performance for all subjects will be assessed: group analysis for mean corrected z-scores (post-training reassessment minus pre-assessment behavioral performance) with non-parametric test will be applied in order to observe performance differences among intervention protocols, as well as longitudinal analysis of the subjects within the groups for Theta and Alpha power values and performance measures for the neurocognitive tasks (accuracy, RTs, block span and mean memory span), during the days of intervention. Later on, we want to analyze the EEG profiles from the subjects, between groups and within groups, to assess intervention effects on EEG phenotypes.

Our hypothesis is that a training protocol with interleaved sessions of NF training and NC tests will have a greater impact on cognitive performance in comparison with either the NF or the NC training alone. By alternating NF training, which provides quantitative measures of the brain state to be modulated, with NC tests, the subject obtains a broader perspective of his own cognitive recovery. Additionally, the subject can adapt his strategy to optimize behavioral performance based on his own EEG state.

As future work, the intent is to develop a rehabilitative intervention, based on the pilot experiment described herein, in order to assess the therapeutic effects of the proposed protocol. This way, it will be possible to verify if the potential effects of intervention are mainly due to a real modulation of the patient brain signals or otherwise, these effects can have other sources such as the stimulation provided by a rich graphic environment or the social contact with clinicians and researchers.

\section{ACKNOWLEDGMENTS}

This work was supported by FCT with the reference project FCOMP 010124 FEDER 021145 (FCT/PTDC/SAUENB/118383/2010). The authors would like to acknowledge the helpful contributions of Ricardo Silva and Nadine Santos to the proposed experiment architecture.

\section{REFERENCES}

[1] B. A. Vicioso, "Dementia: when is it not Alzheimer disease?," Am. J. Med. Sci., vol. 324, pp. 84-95, 2002.

[2] P. M. Rossini, S. Rossi, C. Babiloni, and J. Polich, "Clinical neurophysiology of aging brain: from normal aging to neurodegeneration," Prog. Neurobiol., vol. 83, pp. 375-400, December 2007.

[3] C. Lustig, P. Shah, R. Seidler, and P. a Reuter-Lorenz, "Aging, training, and the brain: a review and future directions," Neuropsychol. Rev., vol. 19, pp. 504-22, December 2009.

[4] K. Ball, D. B. Berch, K. F. Helmers, J. B. Jobe, M. D. Leveck, M Marsiske, J. N. Morris, G. W. Rebok, D. M. Smith, S. L. Tennstedt, F. W. Unverzagt, and S. Willis, "Effects of cognitive training interventions with older adults: a randomized controlled trial," JAMA, vol. 288, no. 18, pp. 2271-2281, 2002.

[5] G. W. Rebok, M. C. Carlson, and J. B. S. Langbaurn, "Training and maintaining memory abilities in healthy older adults: traditional and novel approaches," J. Gerontol. B-Psychol., vol. 62, pp. 53-61, 2007.

[6] J. Dunlosky, A. K. Kubat-Silman, and C. Hertzog, "Training monitoring skills improves older adults' self-paced associative learning," Psychol. Aging., vol. 18, no. 2, pp. 340-345, 2003.

[7] C. Babiloni, F. Babiloni, F. Carducci, F. Cincotti, C. Del Percio, G. De Pino, S. Maestrini, A. Priori, P. Tisei, O. Zanetti, and P.M. Rossini, "Movement-related electroencephalographic reactivity in Alzheimer disease," NeuroImage, vol. 12, pp. 139-46, August 2000

[8] S. Wangler, H. Gevensleben, B. Albrecht, P. Studer, A. Rothenberger, G.H. Moll, and H. Heinrich, "Neurofeedback in children with ADHD: specific event-related potential findings of a randomized controlled trial," Clin. Neurophysiol., vol. 122, pp. 942-950, May 2011.

[9] A. W. Keizer, R. S. Verment, and B. Hommel, "Enhancing cognitive control through neurofeedback: a role of gamma-band activity in 
managing episodic retrieval," NeuroImage, vol. 49, pp. 3404-13, February 2010.

[10] R. C. deCharms, "Applications of real-time fMRI," Nat. Rev. Neurosci., vol. 9, pp. 720-9, September 2008

[11] M. A. Nicolelis, "Actions from thoughts.," Nature, vol. 409, pp. 403-7, January 2001

[12] J. J. Daly, and J. R. Wolpaw, "Brain-computer interfaces in neurological rehabilitation," Lancet Neurol., vol. 7, no. 11, pp. 1032-1043, 2008

[13] R. Coben, and J. R. Evans, Neurofeedback and Neuromodulation Techniques and Applications, Academic Press, 2010.

[14] G. Pfurtscheller, F. H. Lopes da Silva, "Event-related EEG/MEG synchronization and desynchronization: basic principles," Clin. Neurophysiol., vol. 110, pp. 1842-1857, 1999.

[15] M. van Gerven et al., "The brain-computer interface cycle," J. Neural. Eng., vol. 6, no. 4, pp. 1-10, 2009.

[16] L. Maggi, S. Parini, P. Perego, and G. Andreoni, "BCI++: an objectoriented BCI prototyping framework," In Proceedings of the 4th International Brain-Computer Interface Workshop and Training Course, 2008.

[17] P. Sauseng, W. Klimesch, M. Schabus, and M. Doppelmayr, "Frontoparietal EEG coherence in theta and upper alpha reflect central executive functions of working memory.," Int. J. Psychophysiol., vol. 57, no. 2, pp. 97-103, August 2005.

[18] W. Klimesch, "EEG alpha and theta oscillations reflect cognitive and memory performance : a review and analysis,"Science, 1999.

[19] S. T. Mueller, PEBL: The psychology experiment building language (Version 0.12) [Computer experiment programming language]. Retrieved Nov. 2012 from http $/ /$ pebl.sourceforge.net., 2012.

[20] A. C. Paulo et al., "Patterns of cognitive performance in healthy ageing in northern Portugal: a cross-sectional analysis," PloS one, vol. 6, no. 9, pp. e24553, January 2011.

[21] K. Ball, D. B. Berch, K. F. Helmers et al., "Effects of cognitive training interventions with older adults: a randomized controlled trial," JAMA, vol. 288, pp. 2271-2281, 2002.

[22] A. W. Keizer, R. S. Verment, and B. Hommel, "Enhancing cognitive control through neurofeedback: A role of gamma-band activity in managing episodic retrieval," NeuroImage, vol. 49, no. 4, pp. 3404 $3413,2010$.

[23] T. Ros et al., "Mind over chatter: Plastic up-regulation of the fMRI salience network directly after EEG neurofeedback," NeuroImage, vol. 65 , pp. 324-335, January 2013. 